\title{
STUDY OF THE FLUXSET MAGNETIC PROBE SPEED-SENSITIVITY DEVELOPED FOR DETECTION OF MAGNETIC NANOPARTICLES IN SURGERY
}

\author{
Dániel Datz* — Antal Gasparics ${ }^{* *}$ — Gábor Vértesy ${ }^{* *}$
}

\begin{abstract}
Markers for imaging are vital parts of surgical processes. Since radioactive compound pose considerable risk to both the recipient and the surgical staff, alternative marking techniques are of interest. In NANOMAGDYE project, magnetooptically active nano-particles were created for this reason. The detection of the magnetic interaction of low mass nanoparticles in surgical environment is very challenging; so far only quasi-stationary measurements were taken. This paper shows a measurement setup for measurements with continuously moving probe at lower velocities $(5-10 \mathrm{~mm} / \mathrm{s})$ and at hand movement velocity $(20-30 \mathrm{~cm} / \mathrm{s})$. It was found that at the velocity of hand movement there is only probe response amplitude reduction of at most 10-15\% of the original amplitude. These results indicate that the probe used for the detection of the nano-particles is sufficient to be used in practical purposes in surgery.
\end{abstract}

K e y w o r d s: fluxset sensor, nanomagdye, continuous movement, nanoparticles

\section{INTRODUCTION}

In the treatment of cancer, the detection of the sentinel node $(\mathrm{SN})$, which is any node receiving lymph drainage from the tumor site and containing most likely malignancy is an important prognosis step since a positive metastasis histopathological diagnosis requires the complete lymph node chain exeresis. Current methods for labeling the lymph node system use a dye (vital blue) or radio nuclide injection detected through optical or Gamma probes, respectively, or a combination of both types of markers. For instance, radiopharmaceuticals (such as Tc$99 \mathrm{~m}, \mathrm{Tc}-\mathrm{S}$ colloids) are widely used substances as markers in oncologic imaging. Despite their usefulness, they require special care, since they can be dangerous to the recipient and also to the surgical stuff handling the compound [1].

To provide cleaner, less dangerous, but still viable alternatives for substances as markers in oncologic imaging, an EU FP7 collaborative project was performed. The NANOMAGDYE project [2] aimed at developing a novel clinical methodology based on tailored and versatile magnetic and optical nanosystems able to target the sentinel nodes when injected in cancer tumor, and on multimodal detection easy to utilize even with minimum technical requirements for the environment. It consisted in developing tailored and versatile biocompatible nanosystems combining magnetic, optical and/or mechanical properties and in fabricating a new dedicated magneto-optical probe. The spherical particles comprises iron-oxide (maghemite/magnetite shell-core system) core, and Vital Blue dye molecules attached to it through dendritic molecules [3].

Within the project a novel magnetic probe was designed and fabricated [4]. This Fluxset magnetic sensor [5] based probe is capable to detect the presence of magnetic nanoparticles. The magnetic particles to be detected are under the influence of an alternating exciting magnetic field, and the magnetic sensor detects the weak response of particles. The advantages of this approach are mainly related to the high sensitivity of Fluxset probe, to the cost-effectiveness of the detecting instrumentations, and to the ease of use of the probe [6]. It was shown that even $0.7 \mathrm{mg}$ of magnetic nanoparticle of size $30 \mathrm{~nm}$ could be detected from a distance of $3.5 \mathrm{~mm}$ with acceptable signal to noise ratio (Fig. 1).

The test measurements in the project were performed by quasi-stationary movement of the probe over the samples containing magnetic particles. However, for practical use during surgeries, measurements with continuously moving probe must be taken. The purpose of the present work was to study how the magnetic probe performs during its movement. For this reason, alterations in the measurement setup and the controlling program were done and different signal distorting effects were identified. Finally an estimation of the signal distortion caused by the continuous movement of the probe was given.

\section{MEASUREMENTS}

During the measurements the Fluxset probe was moved continuously over a given specimen with different

\footnotetext{
* Pázmány Péter Catholic University, Faculty of Information Technology, Budapest, Hungary, datzdaniel@gmail.com ${ }^{* *}$ Institute of Technical Physics and Material Science, Research Centre for Natural Sciences, HAS, H-1525 Budapest, P.O. Box 49, Hungary
} 


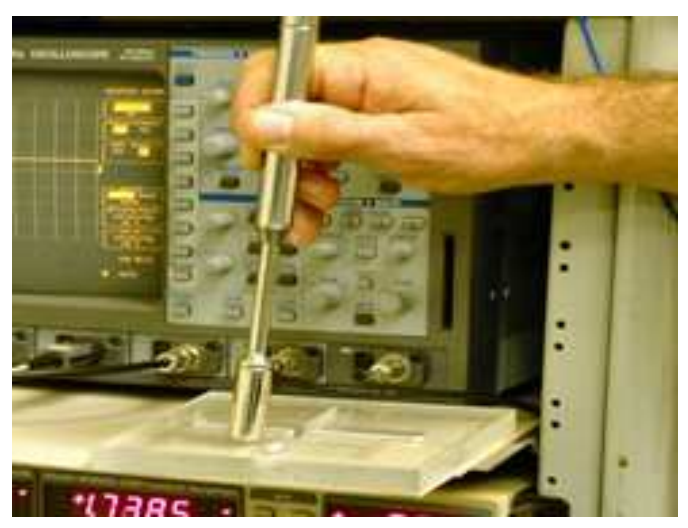

Fig. 1. The functional probe detecting the encapsulated nanoarticles stabilized inside the plastic container
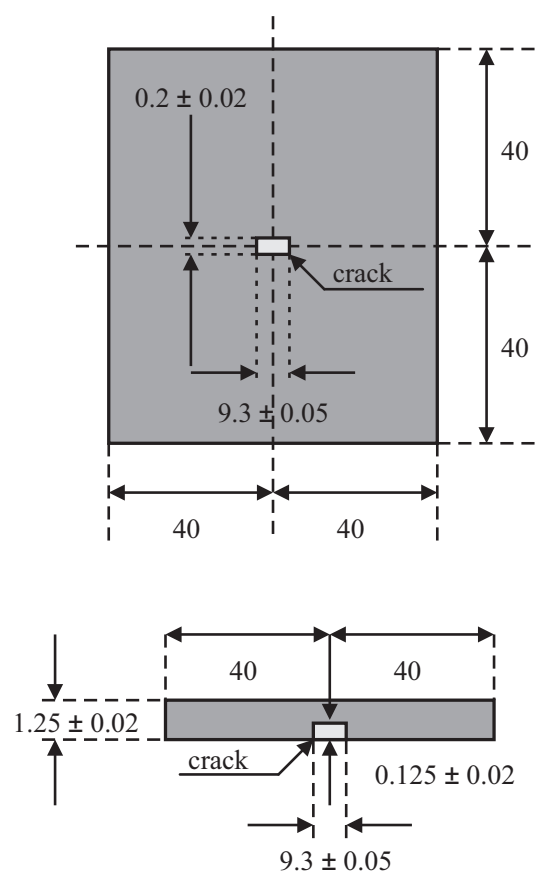

Fig. 2. The geometry of the used aluminum plate with an artificial crack in the centre similar to the known INCONEL-600 benchmark specimen [5]

speeds. In order the emphasize the expected sideeffect of the probe movement a well known and previously many times used eddy current testing configuration with electrically conductive specimen was chosen instead of the magnetic nanoparticles. The specimens were aluminium plates, which contained an artificially made crack. The exciting coil of the Fluxset probe generated eddy currents in the plate and around the crack the eddy currents were distorted, which caused also perturbation of magnetic field around the crack. This perturbation is measured by the sensitive Fluxset sensor. If the probe is scanned along a line over the surface, a typical dipole-character probe response function is obtained, see eg [6], or Fig. 2. Originally this was a step-by-step measurement, but it was found that the physical setup of the Fluxset probe was also capable of continuous measurements. Only the controlling program had to be modified and an digital oscilloscope had to used for the real-time data acquisition, so the other instruments (a controllable table, a lock-in amplifier and function generators) could properly function throughout the measurement.

If the probe is moving continuously over the specimen, two problems can arise. One of them is related to the timing of the measurement devices, where the start causes time-jittering in the recorded signals. In addition the data acquisition and storage during a measurement as the extended measurement time caused inaccuracies in the used instruments. The time-jittering of the signals is caused by the time difference between the start of the movement of the table and the triggering of the oscilloscope dealing with the data acquisition. This time difference is due to the program scheduling of the oprating system that result uncertain time shifts between the issued device commands. The time-jittering could be eliminated by using a simple electronic circuit that triggers the oscilloscope when the table is at a certain position. This way the controlling program only starts the movement of the table, so there is effectively zero time difference between that and the triggering of the oscilloscope.

The oscilloscope periodically needs to be recalibrated. The measurements have to be done in one run to minimize the parameter changes that can effect the signals. Therefore the recalibration of the oscilloscope has to be switched off during the measurement. By taking a high number of measured records with the same parameters, it is found that the lack of calibration has no significant drawback for the precision. The used oscilloscope can store 50000 data points (ie records) which is enough to correctly represent one signal. After taking a signal, the memory of the oscilloscope is transferred to the PC.

Additional distorting effects of the magnetic induction caused by the moving magnetic field above a conducting surface. This is the main subject of our study and this is why ECT setup has been chosen. This problem cannot be ruled out and its effect is estimated based on the measurements, as outlined below. Two cases were studied: movement of the probe with low and with high speed over the specimen. The two cases were investigated by using different experimental arrangements.

\section{RESULTS AND DISCUSSION}

\subsection{Low speed measurements}

Low speed measurements were taken at $5 \mathrm{~mm} / \mathrm{s}$ to $10 \mathrm{~mm} / \mathrm{s}$ speed of the probe over the specimen with $0.5 \mathrm{~mm} / \mathrm{s}$ steps (11 different velocities).

An aluminium plate with an artificial crack (Fig. 2) was used to generate the studied probe response. The excitation signal was $20 \mathrm{kHz}$ sinusoidal signal. 


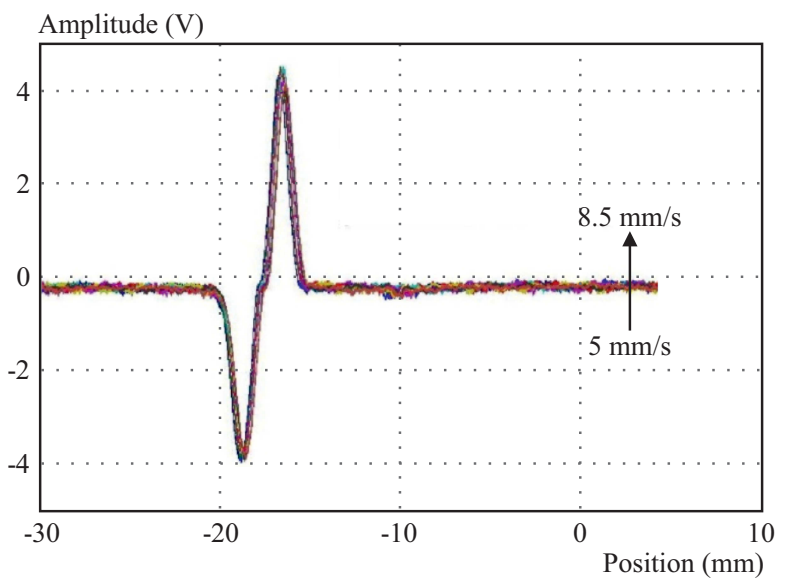

Fig. 3. The signals as a function of the probes position

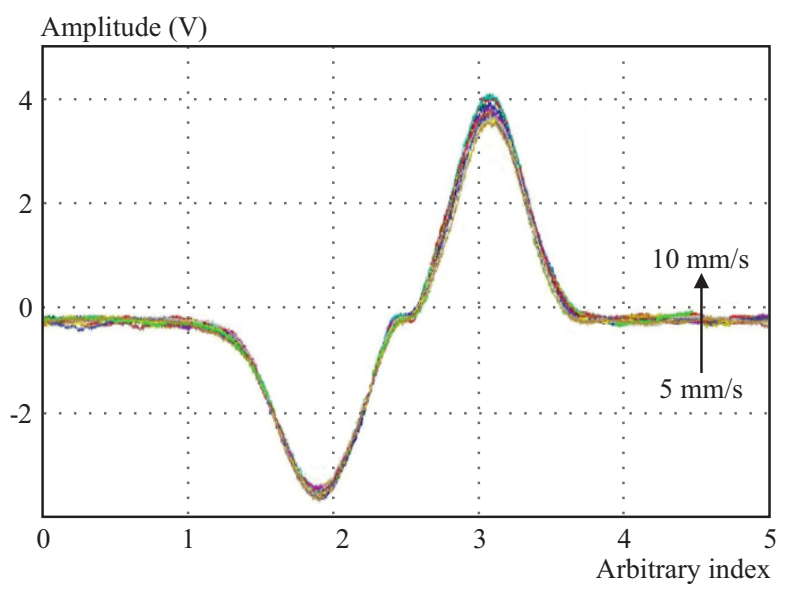

Fig. 4. The relevant parts of the probe response at different velocities cut out and scaled on each other

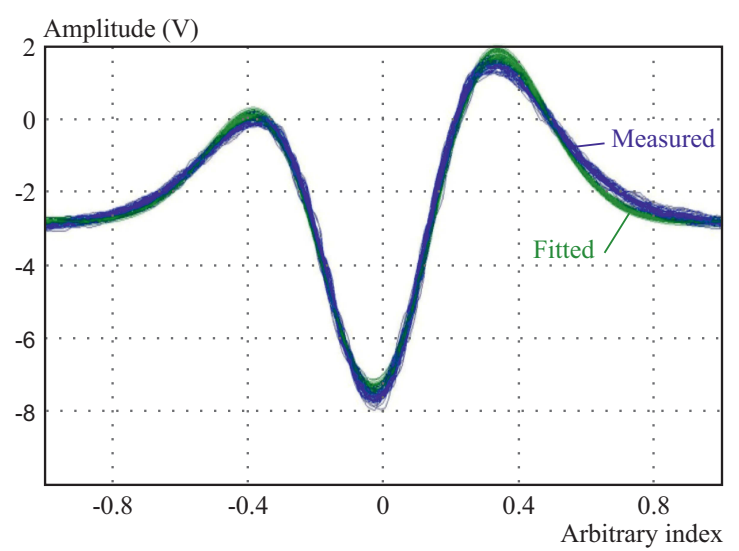

Fig. 5. Cut out and fitted probe responses taken at hand movement velocity scaled on each other

The probe was covered by a thin plastic sheet for protection and pushed directly to the surface of the aluminium plate to maximize the amplitude of the received signal.

The probe response time functions were processed in Matlab. The oscilloscope samples the response of the probe at a constant frequency, meaning the stored data points are equidistant in time, that is the acquired response is a function of time. To acquire the response as a function of the displacement of the probe proper scaling from time to probe position was used. This scaling has to count for the distinct acceleration intervals at the beginning of every measurements. The position at a time $t$ is $s(t)=a / 2 t^{2}$, if $t<t_{m}$ where $t_{m}$ is the time needed to reach the maximal velocity $\left(v_{m}\right)$, or $s(t)=a / 2 t_{m}^{2}+v_{m}\left(t-t_{m}\right)$, if $t>t_{m}$. The functions acquired by this scaling were plotted on each other (Fig. 3). The figure shows that there are no significant distorting effects regarding the position of the responses.

To examine the shape of the responses, $t e^{-t^{2}}$ type functions were fit on the original response functions. The relevant parts were cut out by tresholding the fitted exponential functions and aligned on each other by scaling them on the same interval (Fig. 4). The figure shows that the shape of the responses do not change significantly with velocity.

The results clearly show that there is no significant signal distortion at low velocities.

\subsection{High speed measurements}

For measurements on velocities of the comfortable hand movement speed, different setup with a motor was used with an applied circular aluminum plate $(220 \mathrm{~mm}$ in diameter, $10 \mathrm{~mm}$ width) having the artificial crack ( $2 \mathrm{~mm}$ in diameter, $2 \mathrm{~mm}$ in depth at $75 \mathrm{~mm}$ from centre). The measurement setup was otherwise identical to the one used in low speed measurements.

The motor was designed for much higher velocities and was unable to keep the velocity stable enough in our speed range (this also means the velocity above the crack should be considered as unknown). Also, we had to face with other mechanical difficulties, such as the aluminum plate was not rotating in a perfect plane, thus the probe had to be placed farther from the surface resulting in reduced amplitude. The motorized arrangement gives an impulse (trigger) at the same position at every full rotation. From this impulse the angular velocity could be calculated, but due to the continuous velocity changing that is only the average angular velocity for a whole turn. By constructing a simple circuit that gives an additional trigger impulse and positioning the motor so that, one of the impulses is just right before and the other is just right after the relevant response of the probe, the instantaneous velocity of the motor when the probe is above the crack could be received. Because of the wobbling of the plate the shape of some of the responses are distorted. By taking statistically high amount of measurement, the instantaneous velocities of the probe are placed very densely in a given velocity interval. Since it was seen in the previous measurements, that small differences in velocity did not distort the responses and considering that the effect of magnetic induction is linear with velocity, it can be stated that if the velocity difference of two responses are 

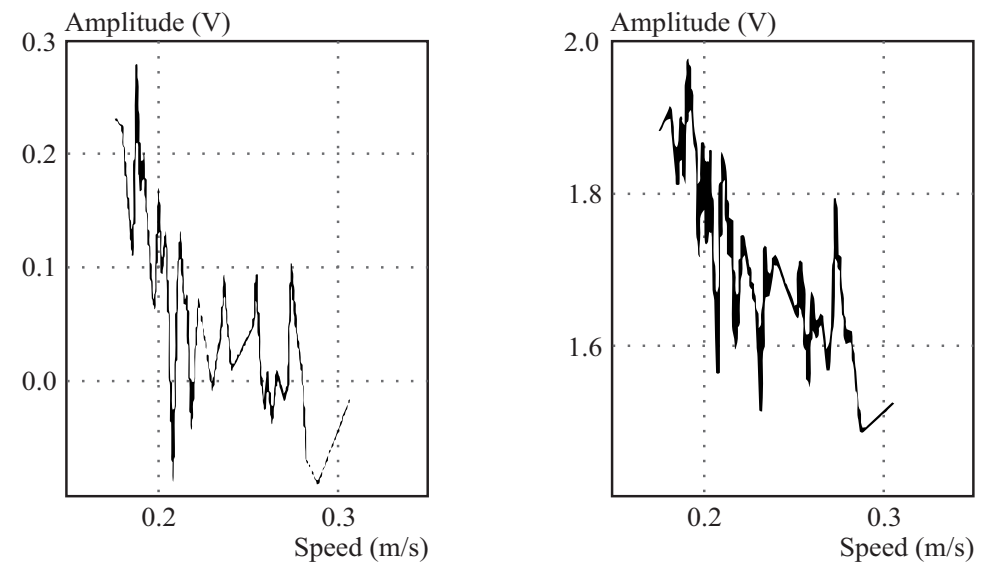

Fig. 6. Amplitudes of the first and second maxima of the analyzed responses. The descending trend is visible

smaller than $2 \mathrm{~mm} / \mathrm{s}$ the responses are qualitatively identical. By dividing the whole velocity interval into numerous $2 \mathrm{~mm} / \mathrm{s}$ wide subintervals, there were 20 such subintervals that contained enough qualitatively identical responses for proper averaging and further work. The signals were processed in Matlab, using the same procedure as at low velocities, now fitting $t^{2} e^{-t^{2}}$ type functions on the responses. The responses at different velocities were cut out and scaled on the same interval together with the fitted functions (Fig. 5). The amplitudes of the first and second maxima of the responses at different speeds can be seen in Fig. 6. It clearly shows a linear descending trend at the amplitude of the maxima of the responses. The change in the amplitude is maximum $10-15 \%$ of the original amplitude.

\section{CONCLUSION}

It was shown that at velocities relevant to surgical use, the signal distorting effects of continuous movement cause amplitude reduction that is at most $10-15 \%$ of the amplitude compared with the no motion case. This effect is much smaller than initially expected and can be easily handled. It means the magnetic probe is fully capable of the measurements during continuous movement on the aluminum plate with a crack. Based on these results, it is expected that similar results will be achieved during the detection of nanoparticles by the moving probe in surgical application, too. A further important problem, which is currently under consideration, is the computerized simulation of the measurements that are presented in the paper.

\section{Acknowledgement}

This paper was supported by the Jnos Bolyai Research Scholarschip of the Hungarian Academy of Sciences.

\section{REFERENCES}

[1] HOOP, B. : The Infiltrated Radiopharmaceutical Injection: Risk Considerations, Journal of Nuclear Medicine 32 (1991), 890-891.

[2] http://nanomagdye.u-strasbg.fr/.

[3] DAOU, T. J.-POURROY, G.-GRENECHE, J. M.-BERTIN, A.-FELDER-FLESCH, D.-BEGIN-COLIN, S. : Water Soluble Dendronized Iron Oxide Nanoparticles, Dalton Trans. (2009), 4442-4449.

[4] GASPARICS, A.-VÉRTESY, G.-PÁVÓ, J.-CACCIOLA, M. : Probe for Detecting Weakly Interacting Magnetic Nanoparticles, International Journal of Applied Electromagnetics and Mechanics 39 No. 1-4 (2012), 29-34.

[5] VERTESY, G.-GASPARICS, A.-SZOLLOSY, J. : High Sensitivity Magnetic Field Sensor, Sensors and Actuators A: Physical 85 No. 1 (Aug 2000), 202-208.

[6] GASPARICS, A.-DARÓCZI, Cs.-VÉRTESY, G.-PÁVÓ, J. : Improvement of ECT Probes based on Fluxset Type Magnetic Field Sensor, in Electromagnetic Nondestructive Evaluation (II) (R. Albanese et al, eds.), IOS Press, 1998, pp. 146-151.

Received 24 May 2014

Dániel Datz born in Budapest, Hungary in 1991. Graduated from Pazmany Peter Catholic University in 2014, he is currently enrolled to a material sciences MSc course at Etvs Lrnd University.

Antal Gasparics (PhD), born in Budapest, Hungary, in 1971. Graduated from Technical University of Budapest in 1995 and received his $\mathrm{PhD}$ degree in 2007. At present he is research fellow of Photonics Department of Hungarian Academy of Sciences, Centre for Energy Research, Institute for Technical Physics and Materials Science of. His main activity field is study of magnetic field sensing and imaging technologies and development of novel nondestructive electromagnetic testing methods.

Gábor Vértesy (DSc, PhD), born in Budapest, Hungary, in 1951. Graduated from Etvs Lornd University, Budapest, in 1974 and received his $\mathrm{PhD}$ degree from Hungarian Academy of Sciences in 1995. At present he is scientific advisor of Photonics Department of Hungarian Academy of Sciences, Centre for Energy Research, Institute for Technical Physics and Materials Science of. His main field is study of ferromagnetic materials and development of novel nondestructive electromagnetic testing methods for characterization of structural industrial materials. 